\title{
Phase I-II study of ADXS31-142 alone and in combination with pembrolizumab in patients with previously treated metastatic castration-resistant prostate cancer (mCRPC): the KEYNOTE-046 trial
}

\author{
Naomi B Haas ${ }^{1 *}$, Mark N Stein ${ }^{2}$, Ronald Tutrone ${ }^{3}$, Rodolfo Perini ${ }^{4}$, Andrew Denker ${ }^{4}$, David Mauro ${ }^{5}$, Lawrence Fong ${ }^{6}$ \\ From 30th Annual Meeting and Associated Programs of the Society for Immunotherapy of Cancer (SITC \\ 2015) \\ National Harbor, MD, USA. 4-8 November 2015
}

\section{Background}

Activation of tumor antigen-specific T-cell responses and bypass/neutralization of tumor immune tolerance mechanisms are essential for immunotherapeutic efficacy. Listeria monocytogenes ( $\mathrm{Lm}$ )-listeriolysin O (LLO) immunotherapies have shown to both generate antigenspecific T-cell responses and neutralize cells that protect the tumor microenvironment. ADXS31-142 is a live attenuated $L m$-LLO immunotherapy designed to target the prostate-specific antigen (PSA) and bioengineered to secrete an antigen-adjuvant fusion protein, consisting of a truncated fragment of the LLO fused to human PSA. Programmed death receptor 1 (PD-1) is a cell surface protein receptor central to $\mathrm{T}$-cell immunity inhibition. Pembrolizumab is a humanized monoclonal antibody that binds to the $\mathrm{PD}-1$ receptor, blocking interaction with PD-1 ligands and thereby inhibiting tumor immune evasion mechanisms. The combination of an $L m$-LLO immunotherapy with an anti-PD-1 antibody has shown synergistic antitumor activity in preclinical studies.

\section{Methods}

This is a Phase I-II, open-label, multicenter, nonrandomized, 2-part study (NCT02325557) in patients with mCRPC. Part A is a dose escalation of monotherapy ADXS31-142, followed by Part B, a dose escalation of ADXS31-142 plus pembrolizumab; Part B is followed by a dose-expansion cohort. Primary objectives are to evaluate the safety and tolerability of ADXS31-142 alone and in combination with pembrolizumab, and establish the ADXS31-142 recommended Phase II dose (RP2D) for monotherapy and combination therapy. Secondary objectives include evaluation of antitumor activity and progression-free survival. Male patients ( $\geq 18$ years) with progressive $\mathrm{mCRPC}(\leq 3$ prior systemic treatment regimens), on androgen deprivation therapy, and with an Eastern Cooperative Oncology Group (ECOG) performance status of $0-1$ are eligible. Each dose-determining part will enroll up to 21 patients. ADXS31-142 and pembrolizumab $(200 \mathrm{mg})$ are administered once every 3 weeks in a 12-week treatment cycle, with ADXS31-142 administration limited to weeks 1, 4, and 7. ADXS31-142 dose-escalations (up to a maximum of $1 \times 10^{10}$ colonyforming units [CFU]) and de-escalations are done according to predefined dose-limiting toxicity criteria by applying the modified toxicity probability interval design. In Part A, the starting ADXS31-142 dose is $1 \times 10^{9} \mathrm{CFU}$, and in Part B is either 1 level below the RP2D established for monotherapy or $1 \times 10^{9} \mathrm{CFU}$, if this was the maximum tolerated dose for monotherapy. Once the ADXS31-142 RP2D for combination with pembrolizumab is determined, the expansion cohort will open for enrollment. A total of 30 patients are planned for treatment at the RP2D. The study is currently open and patient accrual is in progress.

\section{Trial registration}

ClinicalTrials.gov identifier NCT02325557. 


\section{Authors' details}

'Abramson Cancer Center, University of Pennsylvania, Philadelphia, PA, USA.

${ }^{2}$ Rutgers Cancer Institute of New Jersey, New Brunswick, NJ, USA.

${ }^{3}$ Chesapeake Urology Research Associates, Baltimore, MD, USA. ${ }^{4}$ Merck, Sharp

\& Dohme, Corp, North Wales, PA, USA. ${ }^{5}$ Advaxis, Inc., Princeton, NJ, USA.

${ }^{6}$ Department of Medicine, University of California San Francisco Helen Diller

Family Comprehensive Cancer Center, San Francisco, CA, USA.

Published: 4 November 2015

doi:10.1186/2051-1426-3-S2-P153

Cite this article as: Haas et al:: Phase I-II study of ADXS31-142 alone and in combination with pembrolizumab in patients with previously treated metastatic castration-resistant prostate cancer (mCRPC): the KEYNOTE-046 trial. Journal for ImmunoTherapy of Cancer 2015 3(Suppl 2):P153.

Submit your next manuscript to BioMed Central and take full advantage of:

- Convenient online submission

- Thorough peer review

- No space constraints or color figure charges

- Immediate publication on acceptance

- Inclusion in PubMed, CAS, Scopus and Google Scholar

- Research which is freely available for redistribution

Submit your manuscript at www.biomedcentral.com/submit
(Ciomed Central 